\title{
Ulnar Nerve Passing Through Triceps Muscle -Rare Variation
}

\author{
Chandrika G Teli ${ }^{1}$, Nilesh N. Kate ${ }^{2}$, H. S. Kadlimatti ${ }^{3}$ \\ ${ }^{I}$ (Department of Anatomy, ESIC Medical College Gulbarga/ Rajiv Gandhi university of health sciences \\ Karnataka, India) \\ ${ }_{2}^{2}$ (Department of Physiology, ESIC Medical College Gulbarga / Rajiv Gandhi university of health sciences \\ Karnataka, India) \\ ${ }^{3}$ (Department of Anatomy, ESIC Medical College Gulbarga / Rajiv Gandhi university of health sciences
} Karnataka, India)

\begin{abstract}
During routine dissection for undergraduate students, right upper limb of 45 year old male showed variation in the course of ulnar nerve. The ulnar nerve arose as a continuation of medial cord, descended down along medial side of axillary and brachial artery. As the nerve travelled upper third of arm, it pierced the medial head belly of triceps muscle, passing through it for $4-5 \mathrm{~cm}$,emerged out of it to reach near the medial epicondyle. Then the nerve passed behind the medial epicondyle to follow its normal course and distribution. This kind of variation is not been described in the literature.
\end{abstract}

Keywords: lunar nerve, variation in course, entrapment neuropathy.

\section{Introduction}

The ulnar nerve $(\mathrm{C} 7,8, \mathrm{~T} 1)$ is formed from medial cord of the brachial plexus. It lies medial to axillary and brachial artery as far as middle of humerus, and then pierces the medial inter muscular septum to descend on the anterior face of triceps. It passes behind the medial epicondyle to enter the forearm. Here it descends beneath flexor carpi ulnaris until this muscle thins out into tendon, leaving nerve to lie superficially on its radial side. About 2 inches above the wrist, a dorsal cutaneous branch passes deep to flexor carpi ulnaris to supply the dorsal aspects of the ulnar 11/2 fingers and the dorsal aspect or the ulnar side of the hand. Ulnar nerve crosses flexor retinaculum superficially to break up into a superficial terminal branch, supplying the ulnar 11/2 fingers, and a deep terminal branch which supplies hypothenar muscles and intrinsic muscles of hand [1,2].

\section{Case Report}

During routine dissection for undergraduate students, right upper limb of 45 year old male showed following variation in the course of ulnar nerve [figure 1]. The ulnar nerve arose as a continuation of medial cord, descended down along medial side of axillary and brachial artery. As the nerve travelled upper third of arm, it pierced the medial head belly of triceps muscle, passing through it for $4-5 \mathrm{~cm}$,emerged out of it to reach near the medial epicondyle. Then the nerve passed behind the medial epicondyle to follow its normal course and distribution.

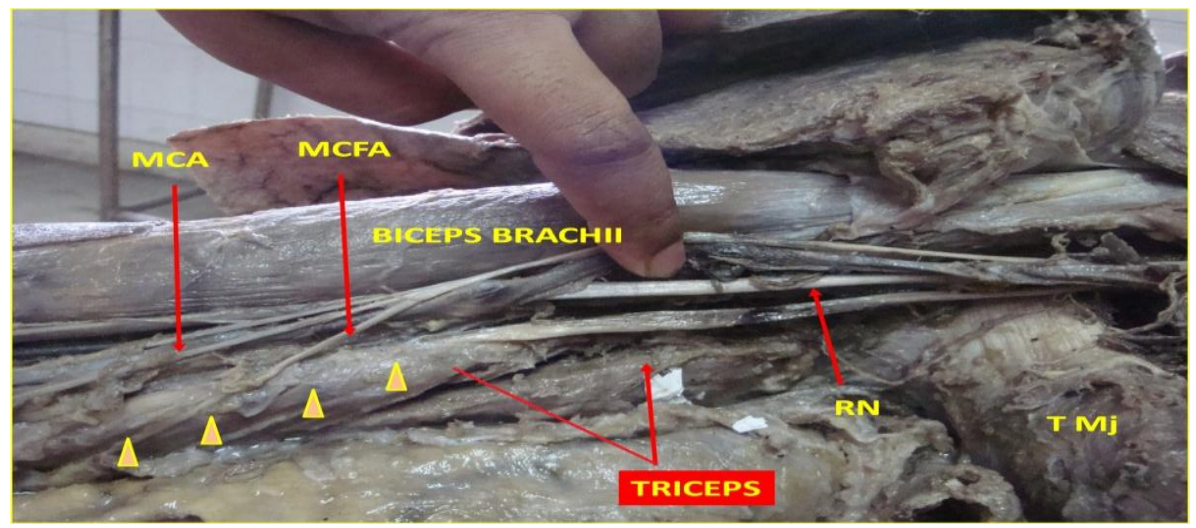

Figure 1: Showing the ulnar nerve [arrow heads]passing through triceps muscle and emerging out near the medial epicondyle. RN-Radial Nerve, MCA-Medial Cutaneous Nerve Of Arm, MCFA - Medial Cutaneous Nerve Of Forearm, $\mathrm{T} \mathrm{Mj}-$ Teres Major. 


\section{Discussion}

The ulnar nerve is in its vulnerable position behind the medial epicondyle of the humerus, may be damaged in fractures or dislocations of the elbow; it is also frequently divided in lacerations of the wrist. In the latter case, all the intrinsic muscles of the fingers (apart from the radial two lumbricals) are paralysed so that the hand assumes the clawed position already described under Klumpke's palsy. The clawing is slightly less intense in the 2nd and 3rd digits because of their intact lumbricals, supplied by the median nerve. In late cases, wasting of the interossei is readily seen on inspecting the dorsum of the hand. Sensory loss over the ulnar $11 / 2$ fingers is present. If the nerve is injured at the elbow, the flexor digitorum profundus to the 4th and 5th fingers is paralysed so that the clawing of these two fingers is less intense than in division at the wrist. Paralysis of the flexor carpi ulnaris results in a tendency to radial deviation of the wrist. Division of the ulnar nerve leaves a surprisingly efficient hand. The long flexors enable a good grip to be taken; the thumb, apart from loss of adductor pollicis, is intact and sensation over the palm of the hand is largely maintained. Indeed, it may be difficult to determine clinically with certainty that the nerve is injured; a reliable test is loss of ability to adduct and abduct the fingers with the hand laid flat, palm downwards on the table; this eliminates 'trick' movements of adduction and abduction of the fingers brought about as part of their flexion and extension respectively.[1]

Typically the ulnar nerve can be compressed in the Cubital tunnel, formed by the tendinous arch connecting the two heads of flexor carpi ulnaris at their humeral and ulnar attachments. Other local causes of compression and neuritis at this site include trauma, inflammatory arthritis, compression by the medial head of the triceps, osteophytes, recurrent subluxation of the nerve across the medial epicondyle of the humerus and abnormal muscular variants such as the anconeus epitrochlearis.

The symptoms are pain at the medial aspect of the proximal forearm together with paraesthesia and numbness of the little finger and ulnar half of the ring finger and the ulnar side of the dorsum of the hand. These symptoms are typically worse on forced elbow flexion. There may also be associated weakness of the muscles of the forearm and the intrinsic muscles of the hand innervated by the ulnar nerve. Interestingly, flexor carpi ulnaris and profundus to the ring and little fingers are frequently spared, presumably because the fascicles supplying these muscles are located on the deep aspect of the nerve. Clawing of the hand is therefore unusual in this syndrome.Surgical treatment involves decompression of the tunnel by division of the aponeurosis of flexor carpi ulnaris with or without subsequent anterior transposition of the ulnar nerve[3].

Two processes account for most instances of ulnar neuropathy at the elbow: compression in the retroepicondylar groove, and compression by the humeroulnar aponeurotic arcade joining the two heads of the flexor carpi ulnaris. While conventional electrodiagnostic criteria may localize an ulnar neuropathy to the elbow, separating retroepicondylar compression from humeroulnar arcade compression is more difficult. In 130 cadaver elbows, the relationships between the medial epicondyle, flexor carpi ulnaris, and ulnar nerve was examined. The humeroulnar arcade lay from 3 to $20 \mathrm{~mm}$ distal to the medial epicondyle, the intramuscular course of the nerve through the flexor carpi ulnaris ranged from 18 to $70 \mathrm{~mm}$, and the nerve exited the flexor carpi ulnaris 28 to $69 \mathrm{~mm}$ distal to the medial epicondyle. In 6 specimens, dense fibrous bands bridged directly between the medial epicondyle and the olecranon proximal to the cubital tunnel proper; accessory epitrochleoanconeus muscles were present in 14 specimens: both may cause ulnar neuropathy at the elbow. Anatomical variations may contribute to the difficulty in separating causes of ulnar neuropathy at the elbow [4].

\section{References}

[1] Ellis Harold in Clinical Anatomy, $11^{\text {th }}$ edition, Blackwell publishing 2006.p. 198 -200

[2] Richard S Snell in Clinical Anatomy by Regions, $8^{\text {th }}$ edition ,Wolters Kluwer Lippincott Williams \& Wilkins; 2012 .p. 338

[3] Harold Ellis, Patricia Collins, David Johnson, Eds. Gray's Anatomy: The Anatomical Basis Of Clinical Practice 39th Ed., London, Churchill Livingstone. 1999; 2024

[4] Campbell WW, Pridgeon RM, Riaz G, Astruc J, Sahni KS 1991Variations in anatomy of the ulnar nerve at the cubital tunnel: pitfalls in the diagnosis of ulnar neuropathy at the elbow. Muscle Nerve. 1991 Aug; 14(8):733-8. 